\title{
La gestión del turismo en los programas de innovación rural de Castilla-La Mancha ${ }^{1}$
}

\section{Tourism management in rural innovation programs of Castilla-La Mancha}

\author{
Águeda Esteban Talaya ${ }^{2}$ \\ Juan Antonio MondéJar Jiménez ${ }^{3}$ \\ JosÉ MondÉJAR JiMÉNEZ ${ }^{4}$ \\ M. ${ }^{a}$ Leticia Meseguer Santamaría ${ }^{5}$ \\ Universidad de Castilla-La Mancha
}

Recibido el 9 de diciembre de 2009, aceptado el 29 de marzo de 2010

$\mathrm{N}^{\circ}$ de clasificación JEL: L83, O18

DOI: $10.5295 /$ cdg.100192ae

Reseña bibliográfica: ESTEBAN, A.; MONDÉJAR, J. A.; MONDÉJAR, J. y MESEGUER, M. L. (2010): "La gestión del turismo en los programas de innovación rural de Castilla-La Mancha", Cuadernos de Gestión, Vol 10, nº especial, pp. 111-124, DOI: 10.5295/cdg.100192ae

\section{Resumen:}

Los programas de innovación rural han supuesto un verdadero revulsivo para determinadas regiones europeas, por cuanto han representado una cuantiosa inversión que ha necesitado de una adecuada gestión para dirigir el destino de unos fondos estructurales. En este sentido, el presente trabajo analiza la inversión de estos programas de desarrollo en medidas de apoyo al sector del turismo rural, que han supuesto un importante incremento de la oferta a diversos niveles: aumento de alojamientos rurales, del número de plazas disponibles para turistas, rehabilitación del patrimonio, puesta en valor de elementos históricos y recuperación, en definitiva, del patrimonio histórico, cultural, arquitectónico y natural del mundo rural.

El trabajo empírico de este estudio se desarrolla para la comunidad autónoma de Castilla-La Mancha, utilizando los últimos datos de inversión disponibles, bajo la metodología Shift-share, que permite identificar los distintos efectos (global, estructural y competitivo) en que podemos descomponer la ejecución financiera de estas medidas.

\footnotetext{
${ }^{1}$ Los autores desean manifestar su agradecimiento a los comentarios recibidos por parte de tres evaluadores anónimos que, sin duda, han contribuido a mejorar la versión final del artículo.

${ }^{2}$ Facultad de Ciencias Jurídicas y Sociales. Cobertizo San Pedro Mártir, s/n - 45.071-TOLEDO

${ }^{3}$ Facultad de Ciencias Sociales.Avenida de los Alfares, 44 - 16.071-CUENCA. Email: JuanAntonio.Mondejar@ $\underline{\text { uclm.es }}$

${ }^{4}$ Facultad de Ciencias Sociales. Avenida de los Alfares, 44 - 16.071-CUENCA

${ }^{5}$ Facultad de Ciencias Económicas y Empresariales.Plaza de la Universidad, 1 - 02.071-ALBACETE.
} 


\title{
Palabras clave:
}

Turismo rural, shift-share, programas de innovación rural.

\begin{abstract}
Rural innovation programs have been a real shock for certain European regions, because they have represented a huge investment that's needed for proper management to direct some structural funds. In this sense, this paper analyzes the investment of these programs in developing measures to support rural tourism sector, which marked a significant increase in supply at various levels: increasing rural accommodation, the number of beds available for tourists, rehabilitation of heritage, value and recovery of historical elements and the historical, cultural, architectural and natural countryside.

The empirical analysis of this study is developed for the region of Castilla-La Mancha, using the latest information available investment under the Shift-share methodology, which identifies the different effects (global, structural and competitive) that these programmes have on these initiatives.
\end{abstract}

\section{Keywords:}

Rural tourism, Shift-share, Rural innovation programs. 


\section{INTRODUCCIÓN}

La Unión Europea ha dirigido buena parte de sus esfuerzos inversores a la fijación poblacional en zonas rurales especialmente desfavorecidas. La Política Agraria Común es, indudablemente, uno de los ejemplos más claros en este sentido. Si bien, la crisis continuada que ha venido registrando este sector en los últimos años resta efectividad a estos planes. Es en este contexto donde surgen los Programas de Innovación Rural (PIR) con el objetivo prioritario de diversificar las actividades rurales, dinamizando el territorio sobre la base de un efecto demostración que permita movilizar la inversión de capital privado con ayuda de inversiones públicas.

El producto turismo rural responde positivamente a todos estos planteamientos anteriores, y se está constituyendo en una alternativa para el desarrollo local del medio rural, por lo que está recibiendo ayudas financieras desde las diversas administraciones públicas y, además, permite disfrutar de un rico patrimonio (Esteban, Mondéjar, Mondéjar y Meseguer, 2008 y 2009).

En este sentido, se debe tener en cuenta que el fuerte dinamismo del sector ha estado relacionado con las nuevas orientaciones dadas por la Unión Europea para el desarrollo de los espacios rurales (LEADER y PRODER), que ha podido llevar a un sobredimensionamiento de la oferta en algunos casos (Chicharro y Galve, 2009), mientras que la región de Castilla-La Mancha no es ajena a este proceso (Cebrián y García, 2009).

Todo ello hace necesario un mayor conocimiento de la demanda y una orientación al cliente, un turista cada vez más exigente, junto a propuestas basadas en la segmentación y especialización (Molina, Martín-Consuegra, Esteban y Díaz, 2007). Se hace evidente la necesidad de configurar la oferta de turismo rural en función de los recursos naturales y culturales que lo distinguen (Moreno, 2002 y Esteban et al., 2008).

En muchas ocasiones, la ausencia de recursos o de fuentes de financiación propias, impiden la materialización de muchos proyectos de especial relevancia para el patrimonio natural y cultural, para la puesta en valor de recursos susceptibles de ser explotados turísticamente e incluso con el fin de recuperar o rehabilitar algunos elementos susceptibles de desaparecer.

En este sentido, es preciso destacar la importancia para las nuevas empresas de desarrollar una actividad turística en el medio rural, que deben hacerla compatible con los principios del desarrollo sostenible, permitiendo así dar respuesta a las expectativas de los nuevos clientes. Asimismo, se trata de iniciativas empresariales puestas en marcha con el apoyo de alguna medida institucional que favorece específicamente las actividades que impulsan el desarrollo endógeno y sostenido, y la conservación del entorno natural (Toledano y Gessa, 2002).

\section{LOS PROGRAMAS DE INNOVACIÓN RURAL EN CASTILLA-LA MANCHA}

En el año 1991 surge la Iniciativa Comunitaria LEADER a propuesta de la Comisión Europea. A esta Iniciativa Comunitaria LEADER I le seguiría el programa LEADER II, que junto al Programa Operativo de Desarrollo y Diversificación Económica de Zonas Ru- 
rales (PRODER), desarrollado por el Gobierno de España para la ejecución presupuestaria, siguiendo la filosofía y metodología LEADER (Esparcia, 2000).

Concretamente, los Grupos de Acción Local (GAL) que gestionan estas iniciativas para el período de programación 2002-2006 en Castilla-La Mancha fueron un total de 29. Así, de todas las iniciativas desarrolladas, 13 corresponden a LEADER + (de un total de 145 a nivel nacional). Por su parte, el Programa PRODER 2 ha sido aplicable a todo el territorio español, con 16 programas en la comunidad autónoma de Castilla-La Mancha (del conjunto de 162 territorios beneficiados por PRODER 2) (Mondéjar, Mondéjar, Vargas y Meseguer, 2008).

De este modo, para este período de ejecución de la Iniciativa Comunitaria LEADER y el Programa de Desarrollo PRODER (LEADER + y PRODER 2), el principal objetivo será activar y consolidar a medio y largo plazo el desarrollo sostenible del espacio rural. Para ello, se establecen diez líneas de inversión en que se dividen las medidas de apoyo imbricadas en la estrategia de desarrollo (Vargas y Mondéjar, 2006).

Teniendo en cuenta las líneas de inversión mayoritarias de apoyo establecidas en la Iniciativa Comunitaria LEADER + y el Programa Operativo PRODER 2, en este trabajo se analiza la línea turismo, una de las mayor inversión. En el cuadro siguiente podemos observar los porcentajes de inversión destinados a las tres principales medidas, a saber: 1.05 (Valorización de productos locales agrarios), 1.06 (PYMES y servicios) y 1.08 (Turismo).

Tabla 1

Distribución porcentual del capital total principales medida

\begin{tabular}{|l|c|c|c|l|c|c|c|}
\hline \multirow{2}{*}{ Comarcas } & \multicolumn{3}{|c|}{ Medidas } & \multicolumn{2}{c|}{ Comarcas } & \multicolumn{3}{c|}{ Medidas } \\
\cline { 2 - 5 } & 1.05 & 1.06 & 1.08 & & 1.05 & 1.06 & 1.08 \\
\hline Sierra Segura & $13,80 \%$ & $27,76 \%$ & $19,34 \%$ & Adiman & $18,23 \%$ & $26,45 \%$ & $19,30 \%$ \\
\hline Campos de Hellín & $20,08 \%$ & $31,27 \%$ & $18,92 \%$ & Alcarria conquense & $14,80 \%$ & $26,45 \%$ & $26,86 \%$ \\
\hline Mancha Júcar & $15,99 \%$ & $38,81 \%$ & $14,44 \%$ & Záncara & $18,11 \%$ & $27,62 \%$ & $19,27 \%$ \\
\hline Manchuela & $17,16 \%$ & $20,26 \%$ & $21,48 \%$ & Adesiman & $10,44 \%$ & $28,43 \%$ & $23,84 \%$ \\
\hline Monte Ibérico & $15,77 \%$ & $20,50 \%$ & $23,55 \%$ & Fadeta & $7,57 \%$ & $27,50 \%$ & $19,86 \%$ \\
\hline Sacam & $14,52 \%$ & $18,92 \%$ & $23,35 \%$ & Molina & $7,90 \%$ & $19,72 \%$ & $28,13 \%$ \\
\hline Montesur & $13,41 \%$ & $28,49 \%$ & $28,23 \%$ & Adel & $7,85 \%$ & $23,01 \%$ & $27,86 \%$ \\
\hline Calatrava & $16,74 \%$ & $16,05 \%$ & $21,81 \%$ & Adac & $5,63 \%$ & $36,01 \%$ & $27,49 \%$ \\
\hline Valle Alcudia & $20,73 \%$ & $14,78 \%$ & $17,34 \%$ & Quijote & $15,07 \%$ & $32,70 \%$ & $14,15 \%$ \\
\hline Guadiana & $13,02 \%$ & $24,11 \%$ & $20,65 \%$ & Adecor & $12,83 \%$ & $33,18 \%$ & $26,84 \%$ \\
\hline Montes Norte & $5,80 \%$ & $16,99 \%$ & $41,37 \%$ & Dulcinea & $12,88 \%$ & $28,56 \%$ & $16,13 \%$ \\
\hline Promancha & $7,89 \%$ & $31,48 \%$ & $16,16 \%$ & Ipeta & $7,81 \%$ & $30,73 \%$ & $19,26 \%$ \\
\hline Tierra libertad & $9,53 \%$ & $22,11 \%$ & $22,92 \%$ & Castillos & $4,57 \%$ & $39,91 \%$ & $23,91 \%$ \\
\hline Cabañeros & $4,86 \%$ & $17,26 \%$ & $21,99 \%$ & Montes Toledo & $9,37 \%$ & $22,06 \%$ & $25,28 \%$ \\
\hline Prodese & $7,66 \%$ & $19,44 \%$ & $36,97 \%$ & Autonómico & $12,16 \%$ & $25,98 \%$ & $23,37 \%$ \\
\hline
\end{tabular}

Fuente: Consejería de Medio Ambiente y Desarrollo Rural (2006). 
A la vista de los resultados anteriores, prácticamente una cuarta parte de toda la inversión en este tipo de programas se realiza en la medida turismo, siendo nueve los Grupos de Acción Local en superar esa cifra (uno de ellos incluso supera el 41\%), situándose la menor inversión en este tipo de medidas en torno al 15\%. No olvidemos que el programa consta de diez líneas instrumentales, dirigidas prioritariamente a: adquisición de competencias, gastos de gestión, administrativos y asistencia técnica, servicios a la población, patrimonio natural, valorización de productos locales agrarios, PYMES y servicios, valorización del patrimonio cultural y arquitectónico, turismo, otras inversiones, formación y empleo (Mondéjar, Vargas y Meseguer, 2009).

\section{INVERSIÓN EN TURISMO}

El turismo constituye, junto con la línea de apoyo a PYMES, los dos ejes mayoritarios para la práctica totalidad de las comarcas (Vargas, Meseguer, Mondéjar y Mondéjar, 2009). A raíz de la intervención de estos programas en el espacio rural, el turismo rural ha sufrido una considerable transformación tanto en el aspecto de la dotación de infraestructuras como en el de la consolidación como una opción destacada dentro del turismo de interior.

\section{Figura 1}

\section{Número de plazas de alojamiento en establecimientos de turismo rural}

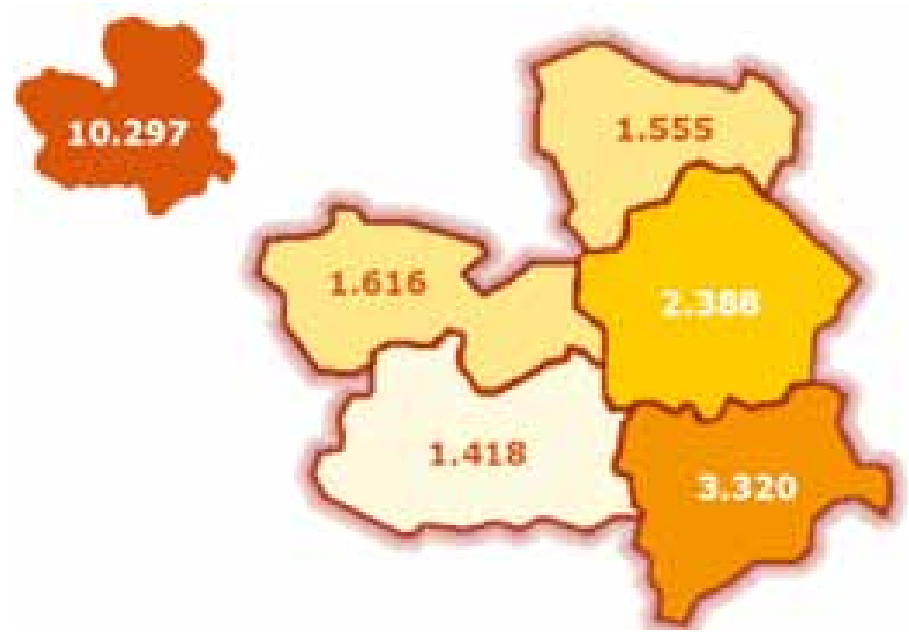

Fuente: Sistema de Investigación Turística de Castilla-La Mancha (2010).

El turismo rural ha experimentado un crecimiento continuo en los últimos años. Es evidente que el fuerte incremento sufrido por la demanda turística ha venido necesariamente acompañado por un aumento de la oferta de alojamientos (su número para el 2010 se muestra en la figura 1) y servicios, con el fin de adecuarse a las exigencias y necesidades del mercado ante un consumidor turístico cada vez más exigente. 
A nivel de oferta, Castilla-La Mancha ha pasado de 352 alojamientos rurales con 2.442 plazas estimadas en el año 2001 (Mondéjar, Mondéjar y Vargas, 2008) a 1.483 establecimientos con 10.297 plazas estimadas en el año 2010 (Sistema de Investigación Turística de Castilla-La Mancha, 2010). De forma análoga, la demanda ha experimentado un incremento notable en los últimos años, llegando a alcanzar 116.858 pernoctaciones en el último trimestre del año 2009.

A continuación se incluyen los coeficientes de localización de la inversión para esta medida específica de turismo: estos coeficientes comparan la proporción de capital invertido por el grupo con la misma proporción en toda la región. Valores superiores a la unidad indicarían una mayor importancia de la medida respecto al resto; por otro lado, valores inferiores a la unidad indicarían una menor repercusión relativa de la medida en el grupo concreto (Vargas, Mondéjar, Mondéjar y Meseguer, 2009).

Figura 2

\section{Coeficiente de localización para Turismo}

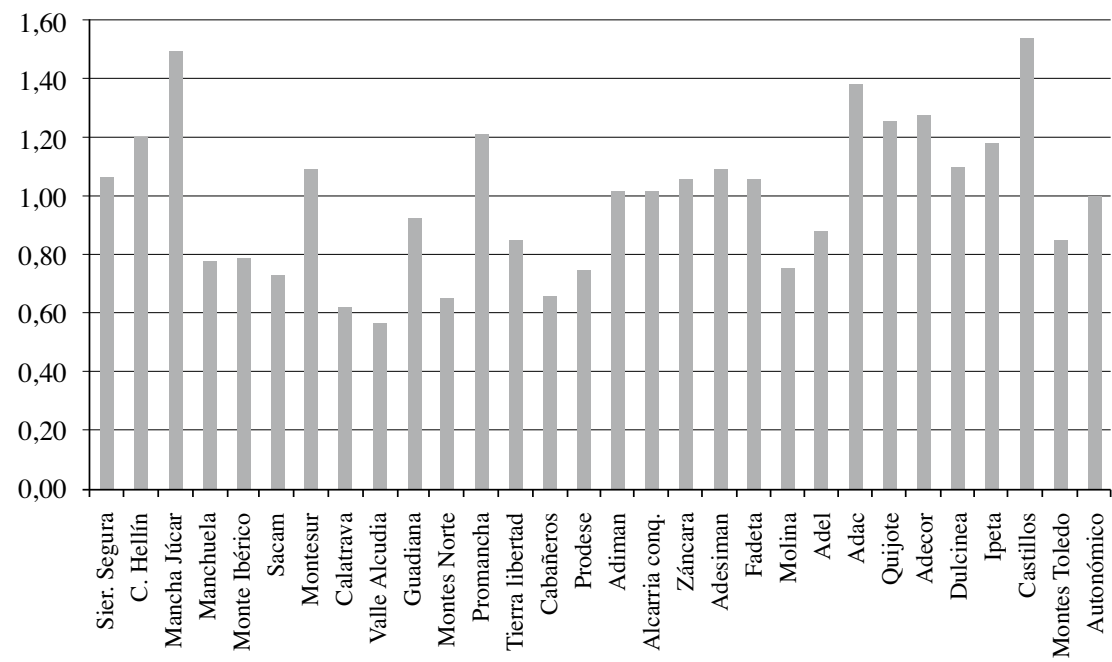

Fuente: Elaboración propia a partir de Consejería de Medio Ambiente y Desarrollo Rural.

Tal y como ponen de manifiesto los datos antecedentes, el conjunto de medidas encaminadas al apoyo a medidas del sector turístico ha supuesto uno de los tres grandes pilares sobre los que se ha sustentado esta política de apoyo a zonas rurales desfavorecidas. Así, junto a las medidas de conservación del patrimonio y la encaminada a favorecer la creación de pequeñas y medianas empresas y servicios en estas zonas, configuran el grueso de los proyectos y actuaciones dirigidas en cada comarca. No obstante, teniendo en cuenta que la generación de capital privado -en términos del grado de ejecución financiera- ha sido menor en el caso de la puesta en valor del patrimonio, es preciso recordar la mayoritaria propiedad de recursos patrimoniales con carácter público, lo que supone una necesidad financiera mayor. 
Figura 3

Grado de ejecución financiera

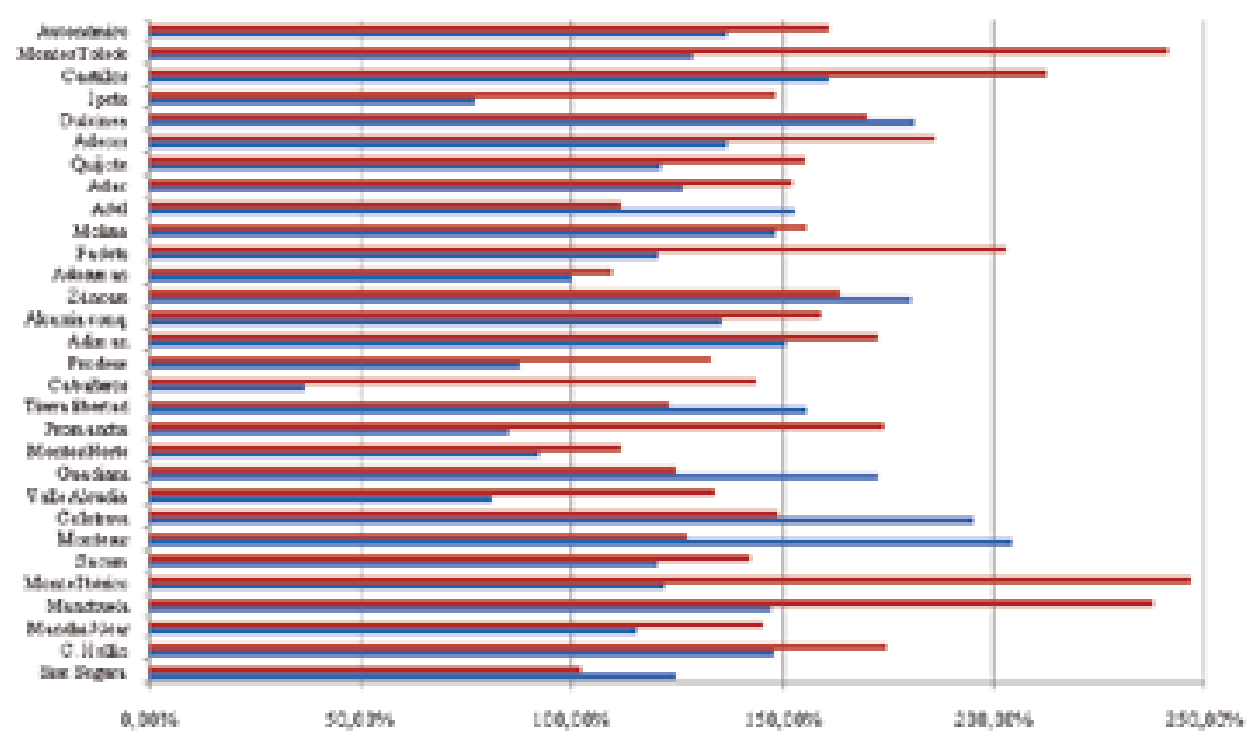

=Capital privado =Capitaltotal

Fuente: Consejería de Medio Ambiente y Desarrollo Rural (2006).

La figura 3 muestra el grado de ejecución financiera de la medida de turismo, considerando la inversión en capital privado y también en capital total: como se pone de manifiesto en el gráfico, los diferentes grupos de acción local han realizado una excelente gestión en esta medida.

Uno de los elementos que ponen de manifiesto la importancia de estas inversiones en turismo rural viene dado por la estructura de la oferta de este producto turístico en CastillaLa Mancha. Si atendemos exclusivamente al número de casas rurales, éstas superan las mil doscientas en el conjunto regional. Teniendo en cuenta su distribución espacial en la comunidad, coincide plenamente con los grupos de acción local más dinámicos en la medida turismo (los cinco grupos que duplican en capital privado).

Para profundizar en el estudio de la capacidad de captación de capital y la distribución espacial del esfuerzo en la inversión turística, se ha optado por realizar un análisis Shiftshare, técnica utilizada en análisis estadístico regional y que permite cuantificar los efectos asociados a la diferente estructura de las comarcas en Castilla-La Mancha y a la "competitividad" de los agentes locales de desarrollo para captar capital privado hacia iniciativas de valorización.

Si denotamos por Kij el capital inicial previsto correspondiente a la medida $\mathrm{i}(\mathrm{i}=1, \ldots$ ,s) para la comarca $\mathrm{j}(\mathrm{j}=1, \ldots, \mathrm{r})$ en el instante inicial y por $\mathrm{K}$ 'ij el capital comprometido en esa medida y comarca en el instante final, entonces la variación experimentada (grado de 
ejecución financiera) puede ser expresada mediante la siguiente identidad (Mayor, López, y Pérez 2005):

$$
K_{i j}^{\prime}-K_{i j}=\Delta K_{i j}=K_{i j} r+K_{i j}\left(r_{i}-r\right)+K_{i j}\left(r_{i j}-r_{i}\right)
$$

donde:

$$
r=\frac{\sum_{i=1}^{q} \Sigma_{j=1}^{r}\left(K_{i j}^{\prime}-K_{i j}\right)}{\sum_{i=1}^{f} \Sigma_{j=1}^{r} K_{\mathrm{i} j}} \quad r_{i}=\frac{\sum_{j=1}^{r}\left(K_{i j}^{\prime}-K_{i j}\right)}{\sum_{j=1}^{r} K_{i j}} \quad r_{i j}=\frac{K_{i j}^{\prime}-K_{i j}}{K_{i j}}
$$

y los tres sumandos en los que se desglosa la variación global de la magnitud estudiada reciben las denominaciones:

$$
\begin{array}{ll}
\text { Efecto Global } & E G_{i j}=K_{i j} r \\
\text { Efecto Estructural } & E E_{i j}=K_{i j}\left(r_{i}-r\right) \\
\text { Efecto Competitivo } & E C_{i j}=K_{i j}\left(r_{i j}-r\right)
\end{array}
$$

Como puede apreciarse en esta descomposición, además de la inercia que supone el efecto global (EG) hemos de considerar las contribuciones al crecimiento (positivas o negativas) derivadas de factores propios de cada ámbito espacial, que vienen recogidas por la suma de Efecto Estructural y Efecto Competitivo, y denominado habitualmente efecto neto. El efecto estructural recoge la influencia positiva o negativa sobre el crecimiento de la distinta importancia relativa de las líneas de patrimonio, con tasas de crecimiento por encima o por debajo de la media regional, respectivamente, pudiendo ser asociado a las posibilidades del patrimonio cultural de cada comarca. Por su parte, el efecto competitivo recoge el especial dinamismo que presenta una comarca respecto al nivel regional en cada línea, por lo que constituiría una medida del "éxito" de la comarca en la captación de capital para la valorización de su patrimonio cultural (Esteban, Mondéjar, Mondéjar y Meseguer, 2008).

Para intentar aislar el efecto de la especialización del efecto competitivo, es frecuente en la bibliografía incluir el llamado "cambio homotético" (Esteban-Marquillas, 1972), que busca la independencia estadística entre ambos efectos y que, en este trabajo, sería el valor que adoptaría el capital previsto en la línea i de la comarca j si la distribución por líneas en esta comarca fuese idéntica a la media autonómica.

Calculando los efectos del modelo Shift-share clásico y agregándolos por comarcas, obtendríamos una estimación de los efectos global, estructural y competitivo, recogidos en el cuadro 2, donde se presenta también la variación real de capital respecto al inicialmente previsto y el grado de ejecución financiera esperado si sólo consideramos el efecto global.

Para una correcta interpretación de la variación real en los capitales previsto y comprometido es necesario en análisis pormenorizado de los efectos estructural y competitivo. Así, el efecto competitivo toma un valor negativo en quince de los veintinueve grupos de acción local, predominando en las provincias de Albacete, Ciudad Real y Toledo. Por su parte, el efecto estructural -de menor cuantía que el competitivo en la mayoría de los grupos- tiene un resultado más heterogéneo por provincias, lo que no permite aclarar patrones de comportamiento, si bien presenta signo positivo en todos los casos. 
Los datos muestran un efecto global especialmente relevante -por encima del millón de euros- en cinco de los grupos de acción local, si bien el cociente de la variación real sobre el efecto global supera la unidad en quince grupos, lo que pone de manifiesto la importancia de esta medida.

Tabla 2

\section{Resultados por grupo del análisis Shift-share}

\begin{tabular}{|c|c|c|c|c|c|c|}
\hline GAL & Provincia & $\begin{array}{c}\text { E. } \\
\text { Global }\end{array}$ & $\begin{array}{c}\text { E. } \\
\text { Estructural }\end{array}$ & $\begin{array}{c}\text { E. } \\
\text { Competitivo }\end{array}$ & Var. Real & $\begin{array}{l}\text { V. Real / } \\
\text { E. Global }\end{array}$ \\
\hline Sierra Segura & Albacete & 552.179 & 189.352 & -239.224 & 502.307 & 0,910 \\
\hline C. Hellín & Albacete & 446.904 & 153.251 & 426.837 & 1.026 .992 & 2,298 \\
\hline Mancha Júcar & Albacete & 554.615 & 190.187 & -646.518 & 98.284 & 0,177 \\
\hline Manchuela & Albacete & 655.851 & 224.903 & 86.112 & 966.865 & 1,474 \\
\hline Monte Ibérico & Albacete & 557.645 & 191.226 & -382.728 & 366.144 & 0,657 \\
\hline Sacam & Albacete & 846.105 & 290.144 & 971.902 & 2.108 .151 & 2,492 \\
\hline Montesur & Ciudad Real & 627.342 & 215.126 & 115.410 & 957.878 & 1,527 \\
\hline Calatrava & Ciudad Real & 480.314 & 164.708 & 495.854 & 1.140 .876 & 2,375 \\
\hline Valle Alcudia & Ciudad Real & 442.725 & 151.818 & -658.387 & -63.843 & $-0,144$ \\
\hline Guadiana & Ciudad Real & 547.747 & 187.832 & -477.158 & 258.421 & 0,472 \\
\hline Montes Norte & Ciudad Real & 1.206 .596 & 413.763 & -1.016 .315 & 604.044 & 0,501 \\
\hline Promancha & Ciudad Real & 349.827 & 119.962 & -184.767 & 285.023 & 0,815 \\
\hline Tierra libertad & Ciudad Real & 589.310 & 202.085 & 350.552 & 1.141 .946 & 1,938 \\
\hline Cabañeros & Ciudad Real & 399.797 & 137.097 & 34.997 & 571.891 & 1,430 \\
\hline Prodese & Cuenca & 1.387.915 & 475.940 & 404.364 & 2.268 .220 & 1,634 \\
\hline Adiman & Cuenca & 656.864 & 225.250 & 154.698 & 1.036 .812 & 1,578 \\
\hline Alcarria conq. & Cuenca & 1.005 .488 & 344.799 & -431.215 & 919.072 & 0,914 \\
\hline Záncara & Cuenca & 714.302 & 244.947 & -311.477 & 647.772 & 0,907 \\
\hline Adesiman & Cuenca & 921.929 & 316.145 & -1.314 .995 & -76.920 & $-0,083$ \\
\hline Fadeta & Guadalajara & 754.329 & 258.672 & 1.696 .280 & 2.709 .281 & 3,592 \\
\hline Molina & Guadalajara & 1.259 .570 & 431.928 & 755.401 & 2.446 .899 & 1,943 \\
\hline Adel & Guadalajara & 1.007 .921 & 345.634 & 695.903 & 2.049 .457 & 2,033 \\
\hline Adac & Guadalajara & 783.155 & 268.558 & -11.744 & 1.039 .969 & 1,328 \\
\hline Quijote & Guadalajara & 359.500 & 123.279 & -237.176 & 245.602 & 0,683 \\
\hline Adecor & Toledo & 639.287 & 219.223 & 219.702 & 1.078 .212 & 1,687 \\
\hline Dulcinea & Toledo & 416.490 & 142.822 & 306.015 & 865.327 & 2,078 \\
\hline Ipeta & Toledo & 646.093 & 221.557 & -931.067 & -63.417 & $-0,098$ \\
\hline Castillos & Toledo & 565.839 & 194.036 & -458.445 & 301.430 & 0,533 \\
\hline Montes Toledo & Toledo & 728.940 & 249.966 & -696.858 & 282.049 & 0,387 \\
\hline
\end{tabular}


Si tenemos en cuenta el cociente entre variación real y efecto global, éste nos muestra valores negativos en tres grupos, donde se ha retrocedido de forma clara en materia turística, lo que pone de manifiesto la apuesta decidida del conjunto de la región por este tipo de medidas.

Para medir la asociación entre comarcas y líneas de financiación, agregando los cocientes entre la especialización de cada comarca en cada línea al cuadrado y el capital homotético, se obtiene el valor de la $\chi^{2}$ de los contrastes de independencia. En este caso, el valor agregado resulta no significativo, por lo que las condiciones de partida de las comarcas no son idénticas, por ello, existen grupos con cierta especialización en algunas medidas (procedente de periodos de programación anteriores) y este patrón permanece.

Por último, si agrupamos a los grupos de acción local según presenten ventajas competitivas o no y si tienen especialización o no, en alguna línea concreta, se puede obtener un cuadro de doble entrada (Alavi y Yasin, 2000), para la medida de turismo (cuadro 3), donde podemos observar la posición de cada uno de los grupos de acción local, clasificados en función de la especialización y la existencia de ventajas de localización. Se considera que hay ventaja o desventaja competitiva dependiendo de si el efecto competitivo es positivo o negativo. Y, a su vez, se habla de especialización en función de si el porcentaje que representa la medida del turismo en cada comarca es mayor o menor que el autonómico (23\%).

Tabla 3

Ventaja frente a especialización en la línea 1.08

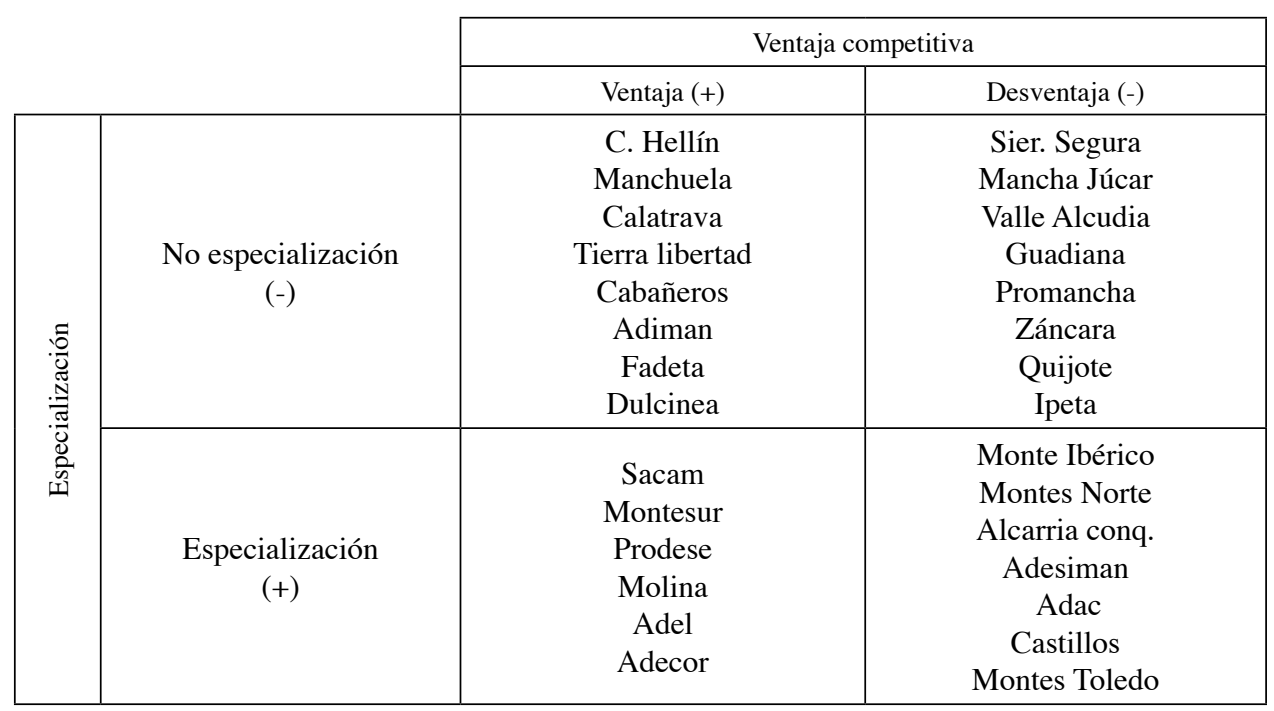

\section{IMPLICACIONES PARA LA GESTIÓN}

El presente trabajo desarrolla un acercamiento a la incidencia de los programas de desarrollo rural en Castilla-La Mancha, concretamente a la tercera iniciativa LEADER + 
y al programa operativo PRODER II, que han cubierto la práctica totalidad del territorio autonómico, tras las experiencias anteriores de LEADER II, PRODER y, con anterioridad, LEADER I (Vargas, Meseguer, Mondéjar y Mondéjar, 2009).

Las áreas rurales de Castilla-La Mancha tienen un verdadero problema de despoblación, en línea con otras comunidades autónomas de nuestro país, donde resulta indiscutible que el principal mecanismo para evitar esta despoblación del mundo rural, la garantía de una renta y nivel de vida de su población comparable en cierta medida con la existente para la población urbana. En este sentido, los programas de innovación rural deben asumir su papel de elemento vertebrador del territorio en aras de suponer una auténtica alternativa a medio y largo plazo para la población rural (Mondéjar, Mondéjar, Monsalve y Vargas, 2007).

Algunos estudios previos habían puesto de manifiesto la importancia de la medida 1.08 de turismo (junto a la 1.06 destinada a inversión en PYMES y servicios). Los datos obtenidos en este nuevo periodo de programación corroboran los estudios anteriores, de tal modo que han sido - una vez más- las que más fondos han concentrado, debido a su capacidad para la movilización de capital privado.

Desde el punto de vista de la gestión, estamos ante un éxito manifiesto, indudablemente representado por los datos que incluye este trabajo: en todos y cada uno de los grupos de acción local se ha invertido más capital privado del inicialmente propuesto, con especial relevancia en cinco de ellos, que han duplicado su asignación inicial.

Si se realiza un análisis de las iniciativas turísticas amparadas bajo estos programas de desarrollo, el resultado nos indica una gran heterogeneidad de proyectos. La variedad de nuevos productos turísticos rurales obliga a los promotores al desarrollo de estrategias novedosas, en función de la disponibilidad de recursos turísticos (de carácter cultural, natural, patrimonial, etc...) existentes en cada comarca. De forma mayoritaria, los proyectos más demandados han sido los alojamientos rurales, convertidos en un verdadero revulsivo para algunas zonas carentes de cualquier posibilidad de pernocta en su territorio.

En un análisis más detallado, el primer comentario ha de ser sobre las diferencias existentes entre comarcas. Naturalmente, los recursos turísticos no están homogéneamente distribuidos, lo que se traduce necesariamente en diferencias de atención por parte de los agentes locales de desarrollo. En este caso, la distribución inicial que los grupos de acción local otorgan al capital previsto responde al grado de conocimiento que éstos tienen sobre las potencialidades del territorio, centrándose en los puntos fuertes de cada comarca. No obstante, existe una apuesta decidida por parte de todos los grupos hacia el sector turístico, que se pone de manifiesto en porcentajes de inversión total situados entre el quince y el cuarenta por ciento.

Como respuesta al esfuerzo inversor de las distintas administraciones públicas, la iniciativa privada ha respondido con un alto grado de implicación, reflejándose en el incremento del capital inicialmente previsto. En este sentido, también aparecen las discrepancias comarcales en este aspecto, centrándose el capital privado en las líneas que ha considerado más atractivas como fuente de rentabilidad.

Para completar esta visión general, la aplicación del análisis Shift-share permite descomponer el grado de ejecución financiera en varios efectos:

Efecto general, asociado al dinamismo de la iniciativa privada hacia el turismo rural en Castilla-La Mancha, como media de los agregados por comarcas y medidas de financiación. 
- Efecto estructural, que se puede asociar a la diferente dotación inicial de patrimonio por comarcas que, por su heterogeneidad, no atrae por igual al capital privado.

- Efecto competitivo, reflejo de las diferencias de captación de capital en cada comarca respecto a la media autonómica en cada medida.

- La situación ideal está conformada por seis grupos de acción local (Sacam, Montesur, Prodese, Molina, Adel y Adecor), que aprovechan su ventaja competitiva especializándose en la medida turismo.

Existen, a su vez, ocho grupos de de acción local que han desarrollado una ventaja competitiva en la línea 1.8 pero que, sin embargo, no consiguen especializarse en la misma, debido a que sus esfuerzos inversores se destinan a otras medidas. Se les recomienda, por consiguiente, especializarse en esta medida, donde además tienen esa ventaja competitiva. No hay que olvidar, además, que algunas de las comarcas tienen numerosos recursos de carácter natural y cultural que coadyuvan a su desarrollo turístico: un ejemplo es la comarca de Cabañeros, cuyo Parque Nacional está considerado como un referente en la Red de Áreas Protegidas de Castilla-La Mancha.

Asimismo, y a pesar de no disfrutar de una ventaja competitiva en este ámbito, siete grupos están especializados en materia turística, mayoritariamente enclavados en zonas montañosas (Monte Ibérico, Montes Norte, Alcarria conquense y Montes de Toledo), de especial relevancia y singularidad para el desarrollo de actividades turísticas. Este hecho viene favorecido, en gran medida, por la mejora de infraestructuras sufrida en la mayoría de las comarcas en los últimos años.

Sin embargo, nueve son los grupos no especializados y que no presentan ventaja competitiva en el ámbito turístico. Es preciso destacar que, algunos de ellos, carecen de la riqueza patrimonial, cultural y paisajística de otras comarcas, mientras que en otros casos se ha producido un efecto demostración derivado de etapas anteriores de los Programas de Innovación Rural: por ejemplo, la comarca albacetense de la Sierra del Segura atesora la mayor concentración de casas rurales en nuestra región y una de las más importantes a nivel nacional, lo que sitúa a algunos de sus municipios dentro del "Top 100" de mayor densidad de establecimientos rurales por localidad.

En definitiva, la principal recomendación en términos de gestión que puede darse a estas comarcas se dirige a seguir trabajando a favor del desarrollo de una ventaja competitiva que sea sostenible en el tiempo y defendible frente a otros productos turísticos, además de encauzar su especialización al ámbito turístico en busca de un doble objetivo de diversificación de sus actividades tradicionales y, también, de rentabilidad para determinadas explotaciones, eminentemente agrarias.

\section{LIMITACIONES Y LÍNEAS DE INVESTIGACIÓN FUTURA}

Es preciso reconocer algunas limitaciones derivadas del propio estudio que, a su vez, posibilitan el desarrollo de nuevas líneas de investigación.

En primer lugar, se trata de un análisis centrado en la región española de Castilla-La Mancha, por lo que es posible dirigir la primera línea de investigación futura hacia otras regiones: inicialmente, las destacadas en importancia a nivel de turismo rural y, en una segunda fase, el total de regiones españolas que trabajan con Programas de Innovación Rural. 
Además, es preciso considerar que los datos considerados en el presente trabajo corresponden a un único periodo de programación, lo que limita la posibilidad de extraer mayores implicaciones desde el punto de vista de la gestión. En este sentido, la consideración de un nuevo periodo de programación - una vez que se disponga de los datos cuantitativos al respecto- contribuirá a la mejora del estudio.

A nivel metodológico, el análisis Shift-Share representa la aplicación de una técnica utilizada en análisis estadístico regional, que permite cuantificar los efectos asociados a la estructura de las comarcas en Castilla-La Mancha, tal y como se ha puesto de manifiesto anteriormente. Sin embargo, es posible que este análisis se complemente con otros posteriores desde el punto de vista de la modelización, teniendo en cuenta la "competitividad" de los agentes locales de desarrollo para captar capital privado hacia iniciativas de valorización.

\section{REFERENCIAS BIBLIOGRÁFICAS}

ALAVI, J. Y YASIN, M. M. (2000): "A Systematic Approach to Tourism Policy", Journal of Business Research, Vol. 48, pp. 147-156.

CEBRIÁN, F. Y GARCÍA, J.A. (2009): "Dimensiones territoriales del turismo rural e Castilla-La Mancha”. Serie geográfica, $\mathrm{N}^{\mathrm{o}} 15$, pp. 79-91.

CHICHARRO, E. Y GALVE, A. (2009): "Alojamientos rurales en España: entre el crecimiento acelerado y el peligro de una sobredimensión”. Serie geográfica, No 15, pp. 125-137.

CONSERJERÍA DE MEDIO AMBIENTE Y DESARROLLO RURAL (2006): Plan Estratégico Regional de Desarrollo Rural, Dirección General de Desarrollo Rural, Junta de Comunidades de Castilla-La Mancha, Toledo.

ESPARCIA, J. (2000): "The LEADER Programme and the Rise of Rural Development in Spain". Sociologia Ruralis, Vol. 40 (2), pp. 200-207.

ESTEBAN, A.; MONDÉJAR, J.A.; MONDÉJAR, J. Y MESEGUER, M.L. (2008): “Análisis de la inversión de los Programas de Innovación Rural en patrimonio cultural como elemento dinamizador del turismo". Revista de Análisis Turístico, Vol. 5, pp. 16-29.

ESTEBAN, A.; MONDÉJAR, J.A.; MONDÉJAR, J. Y MESEGUER, M.L. (2009): “Los programas de innovación rural como elemento dinamizador del turismo". XXI Encuentro de Profesores Universitarios de Marketing Bilbao. Madrid, Esic Editorial.

ESTEBAN-MARQUILLAS, J. M. (1972): "Shift and Share analysis revisited", Regional and Urban Economics, Vol. 2, $\mathrm{N}^{\circ} 3,249-261$.

MAYOR, M. Y LÓPEZ, A. J. (2005): "The spatial Shift-share analysis: new developments and some findings for the Spanish case", Proceedings of the European Regional Science Association ERSA 2005, Amsterdam.

MAYOR, M.; LÓPEZ, A. J. Y PÉREZ, R. (2005): "Escenarios de empleo regional. Una propuesta basada en análisis shift-share", Estudios de economía aplicada, Vol. 23, № 3, pp. 863-887.

MOLINA, A.; MARTÍN-CONSUEGRA, D.; ESTEBAN, A. Y DÍAZ, E. (2007): "Segmentación de la demanda turística: un análisis aplicado a un destino de turismo cultural", Revista de Análisis Turístico, Vol. 4, pp. 36-48.

MONDÉJAR, J.; MONDÉJAR, J. A.; MONSALVE, F. Y VARGAS, M. (2007): "Una propuesta de evaluación del impacto de los programas de desarrollo rural LEADER y PRODER", Ager, Revista de Estudios sobre Despoblación y Desarrollo Rural, Vol. 6, pp. 7-26.

MONDÉJAR, J.; MONDÉJAR, J. A. Y VARGAS, M. (2008): "El turismo rural en cifras: CastillaLa Mancha", TURYDES: Revista de Investigación en Turismo y Desarrollo, Vol. 1, $\mathrm{N}^{\mathrm{o}} 2$, pp. $1-11$. 
MONDÉJAR, J.; MONDÉJAR, J. A.; VARGAS, M. Y MESEGUER, M. L. (2008): "Puesta en valor de recursos rurales en los Programas de Innovación Rural en Castilla-La Mancha", DELOS: Revista Desarrollo Local Sostenible, Vol. 1, No 1, pp. 1-11.

MONDÉJAR, J.; VARGAS, M. Y MESEGUER, M. L. (2009): "Inversión en Turismo y Patrimonio Natural en Castilla-La Mancha”, Revista de Economía, Sociedad, Turismo y Medio Ambiente, Vol. 8-9, pp. 31-46.

MORENO, B. (2002): "Patrimonio Cultural. Puesta en valor y uso. Una reflexión". Vector plus: miscelánea científico-cultural, Vol. 20, pp. 41-49.

SISTEMA DE INVESTIGACIÓN TURÍSTICA DE CASTILLA-LA MANCHA (2010): Boletín Trimestral de Turismo de Castilla-La Mancha, $\mathrm{N}^{\circ} 11$ (cuatro trimestre de 2009). Universidad de Castilla-La Mancha y Junta de Comunidades de Castilla-La Mancha. Disponible en www. turismocastillalamancha.com/investigacion.

TOLEDANO, N. Y GESSA, A. (2002): "El turismo rural en la provincia de Huelva. Un análisis de las nuevas iniciativas creadas al amparo de los programas Leader II y Proder". Revista de desarrollo rural y cooperativismo agrario, Vol. 6, pp. 107-122.

VARGAS, M.; MESEGUER, M. L.; MONDÉJAR, J. Y MONDÉJAR, J. A. (2009): "Los Programas de Innovación Rural en Castilla-La Mancha: un análisis de la inversión en PYMES y servicios", Papeles de Geografía, Vol. 49-50, pp. 161-174.

VARGAS, M. Y MONDÉJAR, J. A. (2006): “Análisis de la inversión de los Fondos Europeos para Desarrollo Rural en Castilla-La Mancha”, CLM.Economía, Vol. 9, pp. 189-238.

VARGAS, M.; MONDÉJAR, J.; MONDÉJAR, J. A. Y MESEGUER, M. L. (2009): "European grants for investment in regional SMEs: the case of Castilla-La Mancha (Spain)", International Business \& Economics Research Journal, Vol. 8, № 4, pp. 85-90. 